\title{
Late Retinal Redetachment Due to Proliferative Vitreoretinopathy after More Than One Decade
}

\author{
Norlelawati $\mathrm{Z}^{1}$, Bastion $\operatorname{MLC}^{2}(\bowtie)$ \\ ${ }^{1}$ Department of Ophthalmology, Faculty of Medicine, Universiti Sains Islam Malaysia, Bandar Baru Nilai, \\ 71800 Nilai, Negeri Sembilan, Malaysia. \\ ${ }^{2}$ Department of Ophthalmology, Faculty of Medicine, Universiti Kebangsaan Malaysia Medical Centre, \\ Jalan Yaacob Latif, Bandar Tun Razak, 56000 Cheras, Kuala Lumpur, Malaysia.
}

\begin{abstract}
A 44-year-old Malay lady presented with recent onset of inferior field defect for a week. Vision was 6/18, N18. She gave a history of myopia and two prior retinal detachment surgeries twelve years previously following which the retina was flat. Subsequently, the vision reduced to hand motions as the macula became involved. She then underwent successful re-attachment vitrectomy surgery for rhegmatogenous retinal detachment due to superior retinal breaks in association with proliferative vitreoretinopathy (PVR) Grade B. We share this interesting case of retinal redetachment in a previously vitrectomised eye that was stable during follow up for more than 10 years. This was associated with late onset PVR. Counselling of patients on the symptoms of redetachment due to late onset PVR is mandatory in patients after retinal detachment surgery.
\end{abstract}

Keywords: Late onset, proliferative vitreoretinopathy, rehabilitation, rhegmatogenous retinal detachment, vitreoretinal surgery

\section{Correspondence:}

Mae-Lynn Catherine Bastion, Department of Ophthalmology, Faculty of Medicine, Universiti Kebangsaan Malaysia Medical Centre, Jalan Yaacob Latif, Bandar Tun Razak, 56000 Cheras, Kuala Lumpur, Malaysia. Tel: +603-91455983 Fax: +60391456673 Email: mae-lynn@ppukm.ukm.edu.my

\section{Introduction}

Proliferative vitreoretinopathy (PVR) is defined as a complex healing process that involves multiple cellular proliferations on the inner and outer retinal surfaces or both,andon vitreous strands which then contract. PVR can occur prior to surgical repair or develop post-operatively to induce either recurrent retinal detachment due to newbreaks or to existing retinal breaks which cannot close or re-open (1). Clinically, PVR is seen as a variety of different intraocular cellular proliferations including epiretinal membranes, subretinal strands, and retinal detachments incombination with star folds, vitreous traction, and anterior loop traction (anteriorPVR) (2). The incidence of PVR ranges from 4-34\% following primary vitrectomy in rhegmatogenous retinal detachment (2). It is the most common and significant cause of delayed failure of primary retinal detachment surgery thus resulting inpoor visual outcome (3).

PVR following vitreoretinal surgery is generally noted to occur between 2 weeks to 45 months. However, $79 \%$ of PVR develops within the first 3 months, and $90 \%$ within the first 6 months. The statistical median time intervals between onset of retinal disease and primary PVR as well as the time intervals between surgery for PVR and the recurrences was noted to be 2 months (2).

Therefore, we would like to describe an unusual case of delayed onset PVR thatresulted in retinal redetachment occurring more than one decade after the primary and secondary reattachment surgeries. It 


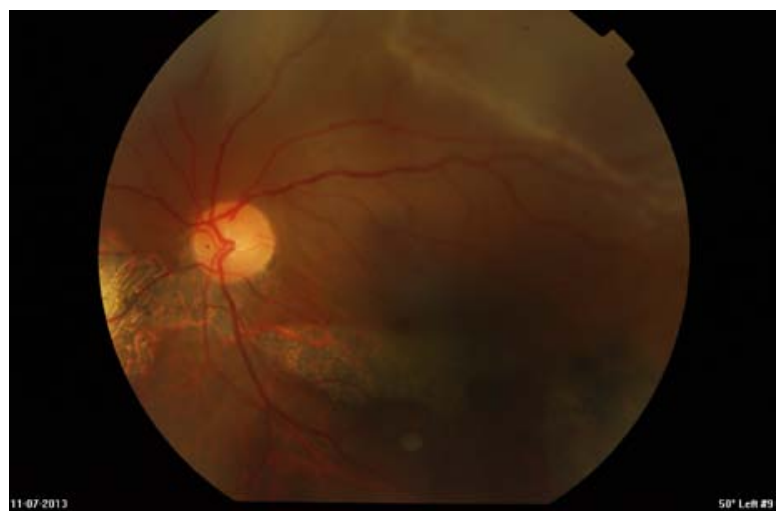

Figure 1: Left fundus photograph showing superior rhegmatogenous retinal detachment involving the macula

emphasizes the importance of patient counselling as to the symptoms of retinal redetachment and the need for immediate presentation even after surgery has already been performed previously.

\section{Case Report}

A 44-year-old Malay lady, a known myope, had left pars planar vitrectomy with gas for rhegmatogenous retinal detachment (RRD) in February 2001. She developed redetachment a month following the surgery in which another surgery was performed with sulphur hexafluoride (SF6) gas tamponade. Her retina was attached until April 2003 when she then underwent uneventful phacoemulsification with intraocular lens implantation. Her post-operative visualacuity was $6 / 18$ with no subsequent symptoms. She then continued regular follow-up at 6 to 9 monthly intervals with no further intervention required for the next 10 years.

She presented to the Eye Casualty in July 2013 with complaint of reduced inferior visual field for 1 week duration. Left visual acuity was 6/18, N18.The inferior visual field defect progressed upward and left visual acuity progressively deteriorated to hand movement within a week. Clinically there was RRD superiorly involving the macula with multiple small holes at the superiorretina. Proliferative vitreoretinopathy Grade B was also observed (Fig. 1).

The patient then had urgent pars planar vitrectomy with drainage retinotomy, heavy liquid fluid exchange, endolaser and SF6 20\% gas as tamponade (Fig. 2). Intraoperatively, it was noted that the original break had sealed and that there was localised superior PVR at the superonasal aspect of the retina in association with residual cortical vitreous which had resulted in new breaks causing a retinal detachment which involved the macula. At 24 months following her third surgery, the retina has remained flat.

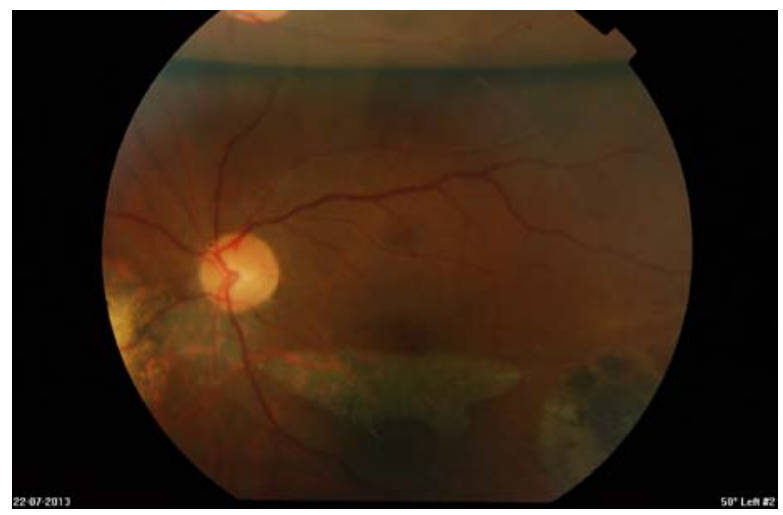

Figure 2: Left fundus photograph showing flat retina two weeks following the vitrectomy with residual gas tamponade superiorly

Her vision was 6/18, N18 unaided same as the visual acuity during follow up prior to the redetachment. There were no issues with intraocular pressure in this patient and treatment was only instituted in the immediate post-operative period at each instance.

\section{Discussion}

Mietz and Heimann investigated the timing of onset of PVR of various etiologies and the timing of onset after interventions. A case of delayed PVR has been reported in a case of severe penetrating ocular trauma occurring after five years. The patient had a history of scleral laceration with uveal prolapse which had not been previously treated (4). The reason for the rarity of late onset PVR is proliferation of the cells is related to a stimulus and is unlikely to start without a cause or precipitant (2). This case and our case which occurred after more than a decade prove to be rare exceptions to the rule.

PVR develops pre-operatively and post-operatively. Pre-operative risk factors for development of PVR include long duration of retinal detachment, large and multiple retinal breaks and concurrent intraocular inflammation or hypoxia. Risk factors for post-operative development of PVR include retained or residual vitreous following the primary operation which is more likely in young individuals with retinal detachment, presence of blood, liberal usage of cryotherapy, traumatic retinal detachment as in this case and persistent intraocular inflammation post-operatively (3).

Various methods have been attempted to overcome PVR including intraocular and systemic steroids, antigrowth factor agents and autologous plasma used intra-operatively but the results remain inconclusive with no single agent able to prevent it (4). The subject has been studied for many years and is still being extensively studied in the laboratory (5). 
Given the discouraging results seen with adjuvants, the importance of thorough vitreous removal particularly during vitrectomy as well as control of inflammation and bleeding to reduce the incidence of PVR subsequently cannot be over emphasized (6). For instance, in this patient contraction of residual vitreous near the superonasal aspect of the vitreous base, a form of localised anterior PVR probably led to late onset retinal tear and detachment. Of interest the location of the tear near the location of the superonasal port highlights the difficulty of viewing and removing vitreous in this location during the initial vitrectomy. Hence the importance of adequate removal of vitreous near the port locations during the first procedure to help reduce the rate of PVR and subsequent redetachment.

With the advance of surgical instrument and techniques, the success rate of reattachment has increased. Nonetheless, the visual results can be disappointing $(6,7)$. Timely reoperation must be the key to retaining vision. Hence, the importance of patient education especially when follow-up many years after the initial surgery would be many months apart or even yearly as illustrated by this case.

Of the treatment options for recurrent RRD, pars planar vitrectomy is the treatment of choice for retinal redetachment secondary to advanced stages of PVR, and may be performed in combination with scleral buckling or with retinectomy in such instances. Scleral buckling alone is limited to cases of mild PVR (6).

\section{Conclusion}

Retinal redetachment may occur in a stable vitrectomised eye after many years. Although this case illustrates that very rarely PVR can occur after more than a decade, regular follow-up is not practical or economical for post-operative retinal detachment patients. Instead, all post-operative patients need to be counselled on the lifelong possibility of redetachment and the symptoms to be anticipated.

\section{References}

1. Leiderman YI, Miller JW. Proliferative vitreoretinopathy: pathobiology and therapeutic targets. Semin Ophthalmol 2009; 24(2): 62-9.

2. Mietz H, Heimann K. Onset and recurrence of proliferative vitreoretinopathy in various vitreoretinal diseases. $\mathrm{Br} \mathrm{J}$ Ophthalmol 1995; 79(10): 874-7.

3. Cardillo JA, Stout JT, LaBree L, et al. Posttraumatic proliferative vitreoretinopathy. The epidemiologic profile, onset, risk factors, and visual outcome. Ophthalmology 1997; 104(7): 1166-73.

4. Waters T,Vollmer L,Sowka J. Proliferative vitreoretinopathy as a late complication of blunt ocular trauma. Optometry 2008; 79(4):197-202.

5. Charteris DG. Proliferative vitreoretinopathy: Pathobiology, surgical management, and adjunctive treatment. $\mathrm{Br} \mathrm{J}$ Ophthalmol 1995; 79(10): 953-60.

6. Moysidis SN, Thanos A, Vavvas DG. Review article: mechanisms of inflammation in proliferative vitreoretinopathy: from bench to bedside. Mediators Inflamm 2012; 2012: 815937.

7. Charteris DG, Sethi CS, Lewis GP, Fisher SK. Proliferative vitreoretinopathy-developments in adjunctive treatment and retinal pathology. Eye (Lond) 2002; 16(4): 369-74. 